\title{
Retraction Note to: An assessment of transgenomics as a tool for gene discovery in Populus euphratica Oliv.
}

\author{
Jing Zhou ${ }^{1} \cdot$ Xin Liu ${ }^{1}$. Shu-Tang Zhao ${ }^{1}$. Jian-Jun Hu ${ }^{1}$. Jie-Wei Zhang ${ }^{2}$ - Jie-Hua Wang ${ }^{3} \cdot$ Xiao-Peng Peng $^{1}$. \\ Xiao-Li Qi ${ }^{1} \cdot$ Tie-Long Cheng ${ }^{1} \cdot$ Meng-Zhu Lu ${ }^{1}$
}

Published online: 26 November 2018

๑) Springer Nature B.V. 2018

\section{Retraction Note to: \\ Plant Mol Biol (2018) 97:525-535 \\ https://doi.org/10.1007/s11103-018-0755-4}

This article (Zhou et al. 2018) has been retracted by the authors because the sequence BIBAC 002A111F06 was incorrectly assigned to the wrong bacterial species. The BIBAC 002A111F06 sequence (GenBank Accession KC129717) reported in the paper was attributed to Populus euphratica Oliv. The BLAST search of this KC129717 sequence against the nr database at NCBI showed that it has very high similarity to a genomic sequence from the gram-negative bacteria Stenotrophomonas maltophilia. The bacterium associates with Populus euphratica Oliv. and DNA isolated from Populus euphratica Oliv. for the construction of the BIBAC clone library inlcuded DNA from
Stenotrophomonas maltophilia. Therefore, the phenotype of the transgenic Arabidopsis line carrying the KC129717 sequence cannot be attributed to genes from Populus euphratica Oliv. The authors apologize for the confusion and misinterpretation of our data resulting from the incorrect sequence assignment. All authors agree to this retraction.

\section{Reference}

Zhou J, Liu X, Zhao S-T, Hu J-J, Zhang J-W, Wang J-H, Peng X-P, Qi X-L, Cheng T-L, Lu M-Z (2018) An assessment of transgenomics as a tool for gene discovery in Populus euphratica Oliv. Plant Mole Biol 97:525-535. https://doi.org/10.1007/s1110 3-018-0755-4
The original article can be found online at https://doi.org/10.1007/ s11103-018-0755-4.

Meng-Zhu Lu

lumz@caf.ac.cn

Jing Zhou

gaha2008@126.com

1 State Key Laboratory of Tree Genetics and Breeding, Research Institute of Forestry, Chinese Academy of Forestry, Beijing 100091, China

2 Beijing Agro-Biotechnology Research Center, Beijing Academy of Agriculture and Forestry Sciences, Beijing 100097, China

3 School of Environmental Science and Engineering, Tianjin University, Tianjin 300072, China 